\title{
Pancreatic sphincterotomy for pancreatitis associated with main duct intraductal papillary mucinous neoplasm
}

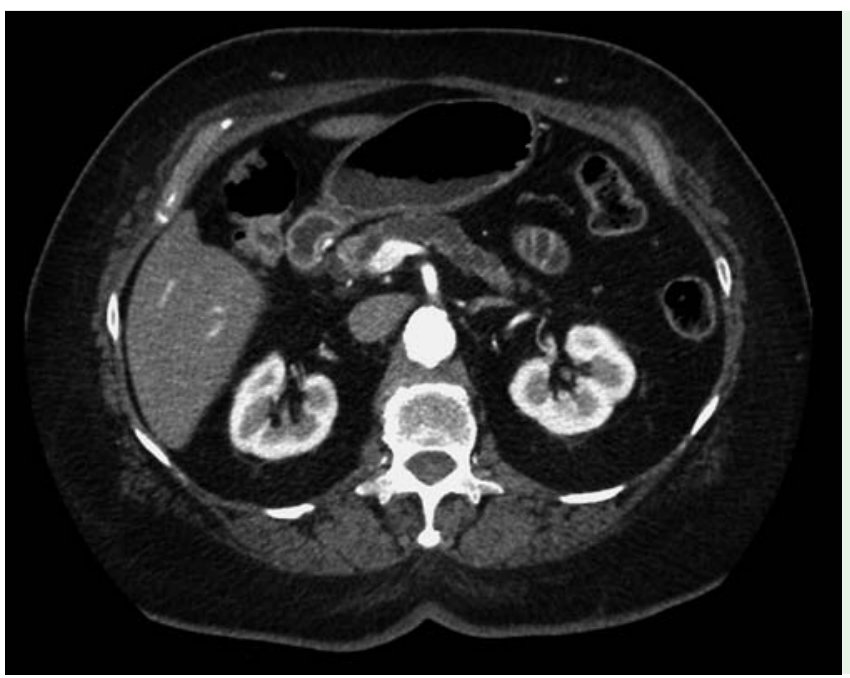

Fig. 1 Abdominal CT with pancreatic protocol demonstrating pancreatic atrophy and diffuse main pancreatic duct dilation.

Recurrent acute or chronic pancreatitis can be presenting symptoms of main duct intraductal papillary mucinous neoplasm (main duct IPMN). Given the risk of malignancy with main duct IPMN, surgery is generally recommended [1]. In patients who are not candidates for surgery, pancreatic sphincterotomy can be considered as a possible way to alleviate pancreatitis due to main duct IPMN by facilitating drainage of mucus.

A 72-year-old woman was evaluated after experiencing two episodes of recurrent pancreatitis in 4 months despite having undergone cholecystectomy. CT scan showed diffuse dilation of the main pancreatic duct and pancreatic atrophy ( Fig. 1).

Endoscopic ultrasonography (EUS) confirmed the CT findings and also demonstrated two dilated side branches of the pancreatic duct. EUS-guided fine-needle aspiration of the larger side branch yielded mucinous material with elevated carcinoembryonic antigen and amylase concentrations. The pancreatogram demonstrated diffuse dilation of the main pancreatic duct with hypoechoic filling defects ( $\bullet$ Fig. 2).

Pancreatic sphincterotomy was performed and the patient has not had recurrent pancreatitis in 6 months.

A 52-year-old man with multiple myeloma was evaluated because he was experien- cing weekly episodes of pancreatitis despite having undergone cholecystectomy for cholelithiasis. Endoscopic retrograde cholangiopancreatography (ERCP) demonstrated a patulous major papilla and mild dilation of the main pancreatic duct in the head. Pancreatic sphincterotomy was performed without incident and the patient did well for approximately 2 years before having recurrent pancreatitis. Repeat ERCP showed dilation of the main pancreatic duct in the head and uncinate process, and a balloon sweep of the pancreatic duct yielded several mucus plugs. The patient had one episode of pancreatitis several months after the last ERCP, which was managed on an outpatient basis. He had no additional episodes of pancreatitis afterwards, and died 6 years after pancreatic sphincterotomy from multiple myeloma.

Complete resolution of recurrent pancreatitis due to main duct IPMN after pancreatic sphincterotomy alone has been reported previously in three patients $[2,3]$. Our series reports the longest follow-up in the literature after pancreatic sphincterotomy for main duct IPMN.

\section{Endoscopy_UCTN_Code_TTT_1AR_2AI}

Competing interests: Dr. Dua is a consultant for Boston Scientific Corporation.

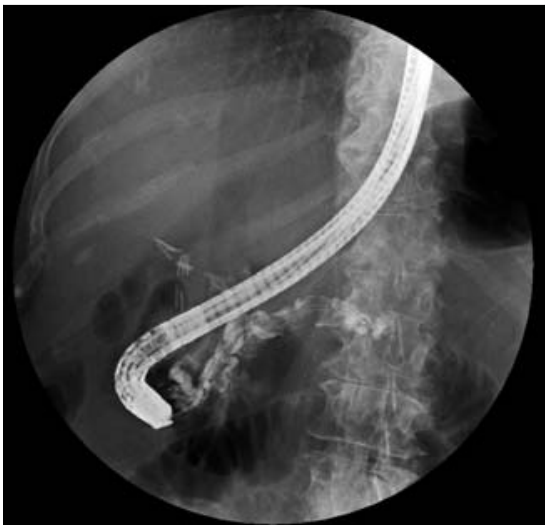

Fig. 2 Pancreatogram showing diffuse dilation of the main pancreatic duct with multiple hypoechoic filling defects representing mucin.

\section{Y. S. Oh, K. Dua}

Division of Gastroenterology and Hepatology, Medical College of Wisconsin, Milwaukee, Wisconsin, USA

\section{References}

1 Serikawa M, Sasaki T, Fujimoto Y et al. Management of intraductal papillary-mucinous neoplasm of the pancreas: treatment strategy based on morphologic classification. J Clin Gastroenterol 2006; 40: 856 - 862

2 Elton E, Howell DA, Parson WG et al. Endoscopic pancreatic sphincterotomy: indications, outcome, and a safe stentless technique. Gastrointest Endosc 1998; 47: $240-$ 249

3 Nocente R, Gentiloni N, Gasbarrini A et al. An apparent idiopathic case of relapsing acute pancreatitis. Hepatogastroenterology 2001; 48: $572-573$

Bibliography

DOI $10.1055 / \mathrm{s}-0030-1255799$

Endoscopy 2011; 43: E75

(c) Georg Thieme Verlag KG Stuttgart · New York . ISSN 0013-726X

\section{Corresponding author}

\section{Y. S. Oh, MD}

Division of Gastroenterology and Hepatology

Medical College of Wisconsin

9200 W. Wisconsin Ave.

Milwaukee, WI 53226

United States of America

Fax: +1-414-955-6214

yoh@mcw.edu 